INRA Prod. Anim., 2004, 17 (1), 3-10
B. MICHALET-DOREAU ${ }^{\dagger 1}$,

F. CORNELOUP ${ }^{1}$, B. AIZAC ${ }^{2}$, J. ANDRIEU ${ }^{1}$, R. BAUMONT ${ }^{1 *}$

${ }^{1}$ INRA, Unité de Recherches sur les Herbivores, Theix, F-63122 St Genès Champanelle

${ }^{2}$ INRA, Domaine du Magneraud, F-17\%00 St Pierre d'Amily

* Correspondance :

baumont@clermont.inra.fr

\section{Variabilité et} facteurs de variation de la teneur en matières azotées des maïs récoltés en plantes entières
La plante de maïs se caractérise par une valeur énergétique et une ingestibilité élevées, et c'est un fourrage facile à conserver sous forme d'ensilage. Ces trois atouts font du maïs ensilage un fourrage intéressant pour alimenter les animaux dont les besoins de production sont élevés. Aussi est-ce encore maintenant la principale source de fourrage dans l'alimentation hivernale des ruminants laitiers et des taurillons à l'engrais. En revanche, la valeur azotée du maïs est faible du fait de sa faible teneur en matières azotées totales (MAT). Avec l'interdiction des farines animales et la crainte des consommateurs face à l'utilisation de tourteaux de soja génétiquement modifiés, la question des ressources en protéines en alimentation animale se pose de façon plus cruciale. Compte tenu

\section{Résumé}

Cette synthèse a pour objectif de faire le point sur les différents facteurs de variation de la teneur en matières azotées totales (MAT) du maïs plante entière, au moment de la récolte et après ensilage, à partir d'une banque de données (16 368 parcelles de maïs plante entière sur pied) constituée des résultats d'essais conduits par le GEVES en vue de l'inscription de nouvelles variétés de maïs au catalogue officiel, et des données de la littérature. Dans cette banque de données, la teneur en MAT des maïs sur pied est en moyenne de $7,7 \pm 0,7 \%$ MS et varie entre 4,7 et $10,5 \%$ MS. Elle est liée à la teneur en matières minérales, ce qui traduit le fait que l'absorption de l'azote est étroitement liée à celle des éléments minéraux. La teneur en MAT des maïs a tendance à diminuer avec le stade de maturité, de 0,20 point entre le stade pâteux-vitreux et le stade vitreux dur. La variabilité due aux conditions de milieu est supérieure à la variabilité due à la variété, soit un écart type moyen de 0,65 point et de 0,47 point respectivement. La relation négative observée dans un certain nombre d'analyses entre la teneur en MAT et le rendement traduit un effet négatif indirect de la sélection des maïs sur leur teneur en MAT, mais cette relation n'est pas systématique. Le processus d'ensilage entraîne une augmentation passive de la teneur en MAT de 0,40 point en moyenne avec de profondes modifications de la composition biochimique des protéines. du contexte économique actuel, la faible teneur en MAT des ensilages de maïs constitue un handicap de plus en plus lourd dans l'alimentation des ruminants. De plus, depuis quelques années, les éleveurs rapportent une diminution des teneurs en MAT des maïs. Cette diminution pourrait être la résultante d'un effet de dilution des matières azotées en relation avec une augmentation des rendements, d'un effet de la sélection qui aurait favorisé indirectement des hybrides à faible teneur en MAT mais ayant un rendement élevé, ou encore une conséquence des modifications des conditions de culture (irrigation, fertilisation ...). Pour tenter de répondre à ces questions, un gisement d'informations est disponible au GEVES (Groupement d'étude et de contrôle des variétés et des semences) qui dispose d'une importante banque de données, constituée des résultats d'essais conduits en vue de l'inscription de nouvelles variétés de maïs au catalogue officiel.

Cette synthèse fait le point sur les différents facteurs de variation de la teneur en MAT du maïs plante entière, au moment de la récolte et après ensilage, à partir de cette banque de données, et des données de la littérature pour les facteurs de variation qui ne pouvaient pas être étudiés à partir de la banque de données du GEVES.

\section{1 / Description de la base}

La banque de données utilisée compte 16368 parcelles de maiis plante entière sur pied. Elle regroupe cinq années d'expérimentation, de 1996 à 2000. Les fourrages, cultivés sur des micro-parcelles de $8 \mathrm{~m} 2$, correspondaient à 
plus de 500 hybrides différents. La majorité des essais était localisée sur les départements du Nord et Nord-Est de la France, et dans le grand Ouest (figure 1), c'est-à-dire essentiellement dans des zones où les températures estivales sont modérées et la pluviométrie rarement limitante. Au sein de chaque essai, une trentaine de variétés était testée selon un dispositif en blocs avec trois répétitions.

Cette banque associe des données sur le rendement (en t MS/ha), et des données sur la composition chimique des maïs à la mise en silo ainsi que leur digestibilité enzymatique (Aufrère et Michalet-Doreau 1988), estimées par spectrométrie dans le proche infrarouge. Nous avons conservé uniquement les fourrages (13 310 fourrages ) correspondant à un stade de récolte classique, soit une teneur en matière sèche (MS) comprise entre 28,5 et $40 \%$.

Figure 1. Répartition géographique des essais.

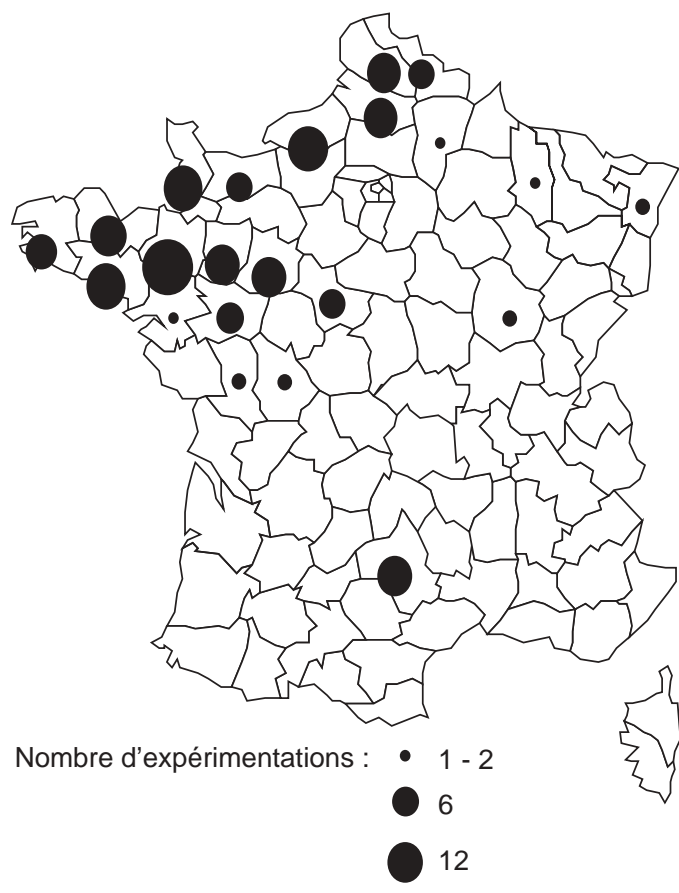

Figure 2. Distribution des teneurs en MAT du maïs plante entière au moment de la récolte (données du GEVES entre 1996 et 2000).

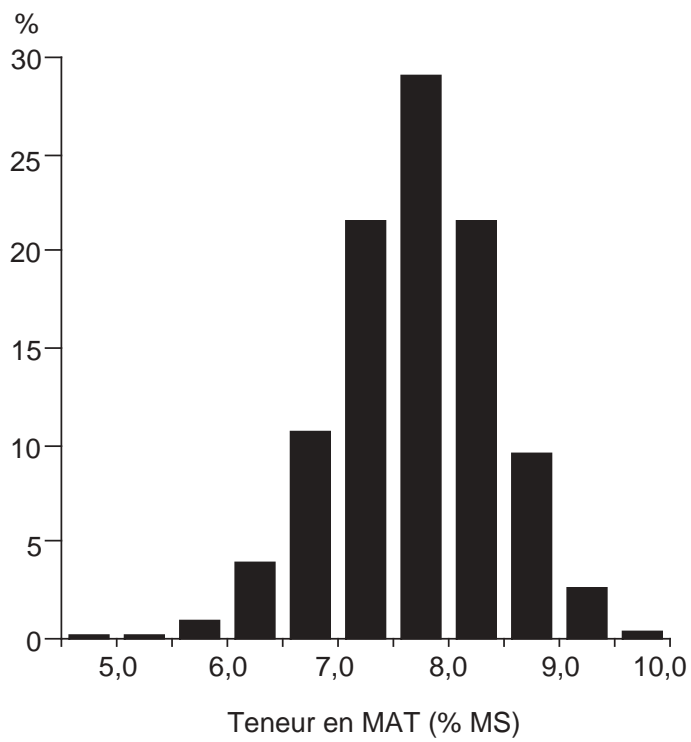

La teneur en MAT des maïs au moment de la récolte est en moyenne de 7,7\%MS, soit une valeur très proche de celle obtenue par Andrieu et al (1993) sur 234 données issues du « club digestibilité » $(7,4 \% \mathrm{MS})$. Létendue de la plage de variation est importante, de 4,7 à $10,5 \% \mathrm{MS}$, et la moitié des données a une teneur en MAT comprise entre 7,2 et 8,2 \% MS (figure 2).

Comme pour d'autres caractéristiques du maïs, la teneur en MAT de la plante est la résultante des effets des facteurs intrinsèques à la plante (stade de végétation, variété) et des conditions de milieu (lieu et année). Pour étudier les effets respectifs de ces deux grands types de facteurs sur la teneur en MAT des maîs, nous les avons isolés au sein de sous-ensembles homogènes. Pour évaluer les effets du génotype, nous avons regroupé les données correspondant à un même milieu, celui-ci étant défini comme une même grande parcelle récoltée une année donnée et conduite de façon identique sur toute sa surface. Nous avons ainsi sélectionné 203 sousensembles, désignés sous le terme de sous-ensembles « variété », correspondant chacun à un milieu donné et regroupant au moins 10 variétés de maïs différentes. De la même manière, pour évaluer les effets du milieu, nous avons regroupé les données par variété, obtenant ainsi 23 sous-ensembles, nommés sousensembles « milieu », regroupant au moins 20 milieux différents par variété.

\section{2 / Teneur en MAT et stade de maturité}

Sur l'ensemble des données de la banque, la teneur en MS des maïs étudiés est en moyenne égale à 33,2\%MS, et se répartit de manière équilibrée entre les stades pâteux-vitreux (30\%), vitreux (33\%) et vitreux dur (37\%). La teneur en MAT des maïs a tendance à diminuer avec le stade de maturité, mais cette diminution est faible, 0,2 point entre le stade pâteux-vitreux $(7,8 \% \mathrm{MS})$ et le stade vitreux dur (7,6 \%MS). Ainsi, les teneurs en MS et en MAT sont significativement liées $(R=-0,141$, $\mathrm{N}=13310$ ). Mais cette relation est obtenue avec des variétés différentes cultivées dans des milieux différents, ce qui rend difficile l'interprétation des résultats. La banque de données dont nous disposions ne nous permettait pas de séparer les effets du stade de végétation des effets de la variété ou du milieu, aussi nous avons étudié l'effet strict du stade de maturité à travers les résultats de la littérature, en suivant l'évolution de la teneur en MAT d'une même variété de maïs dans un même milieu.

Entre $25 \%$ MS et $40 \%$ MS dans la plante entière, la teneur en MAT de la plante sur pied ou après ensilage reste relativement stable (Huber et al 1965, Johnson et McClure 1968, Montgomery et al 1974, Wilkinson et Phipps 1979) ou diminue très lentement (Bal et al 1997, Phipps et al 2000). Nous avons analysé l'évolution de la teneur en MAT de maïs plantes entières en fonction de leur teneur en MS sur 4 hybrides récoltés en 1987 et 1988 en 
quatre lieux différents (figure 3). La teneur en MAT diminue avec l'augmentation de la teneur en MS dans la plante, et cette courbe de diminution s'ajuste à un modèle curvilinéaire de la forme :

MAT $=$ lieu $+\mathrm{b} / \mathrm{MS}^{2}$

$(\mathrm{N}=35, \mathrm{R}=0,96)$

A partir de cette courbe, il a été possible de calculer la diminution moyenne de la teneur en MAT entre 30 et $37 \%$ MS, soit une plage de variation comparable à celle de la banque de données précédente, indépendamment du lieu et de l'année d'expérimentation. Ainsi nous observons que la teneur en MAT diminue de 7,6 à $7,3 \%$, soit 0,25 point contre 0,20 point dans la banque de données du GEVES.

Entre les stades laiteux et vitreux, la teneur en MAT des organes de la plante, tiges, grains, spathes et rafles, évolue peu (figure 4). Seule la teneur en azote des feuilles diminue sensiblement, de $13,5 \%$ à $8,5 \%$ (Aerts et al 1976). La contribution du grain à la fraction azotée totale de la plante augmente notablement à l'approche de la maturité du fait de l'augmentation de la part du grain, mais également de la faible teneur en MAT des fractions végétatives. A une teneur en MS de la plante entière voisine de $35 \%$, les matières azotées du grain représentent $65 \%$ des matières azotées totales de la plante (figure 5). Ces matières azotées du grain sont majoritairement composées de protéines, principalement des zéines et des glutélines, qui sont peu solubles et donc lentement dégradables dans le rumen.

\section{3 / Teneur en MAT et variété}

Nous avons étudié l'effet de la variété dans les 203 sous-ensembles « variété » constitués à partir de la banque de données. Cet effet est significatif dans plus de $80 \%$ des analyses et explique en moyenne $65 \%$ des variations de teneur en MAT des plantes de maïs au sein d'un même milieu. Mais la variabilité induite par la variété reste néanmoins relativement faible, l'écart type moyen pondéré lié à la variété étant en moyenne de 0,47 point. Cet

Figure 3. Effet des conditions de milieu (lieu $x$ année) et du stade de végétation sur la teneur en MAT du maïs plante entière au moment de la récolte (d'après Andrieu et al 1993).

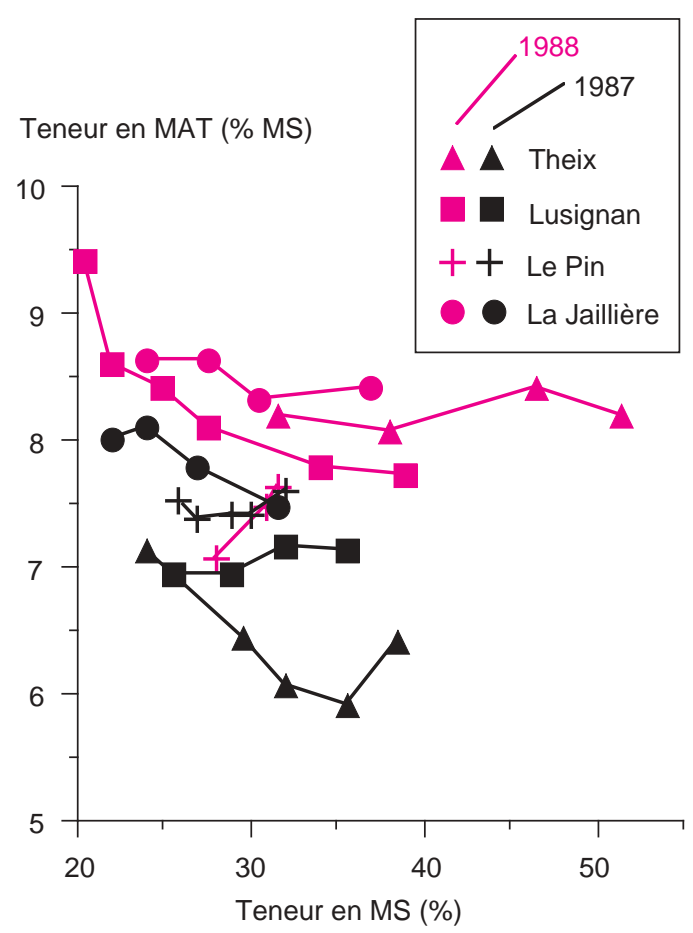

La teneur en MAT des maïs diminue avec l'avancement de la maturité.

Figure 4. Evolution de la teneur en MAT dans les différents organes du maïs (d'après Aerts et al 1976).

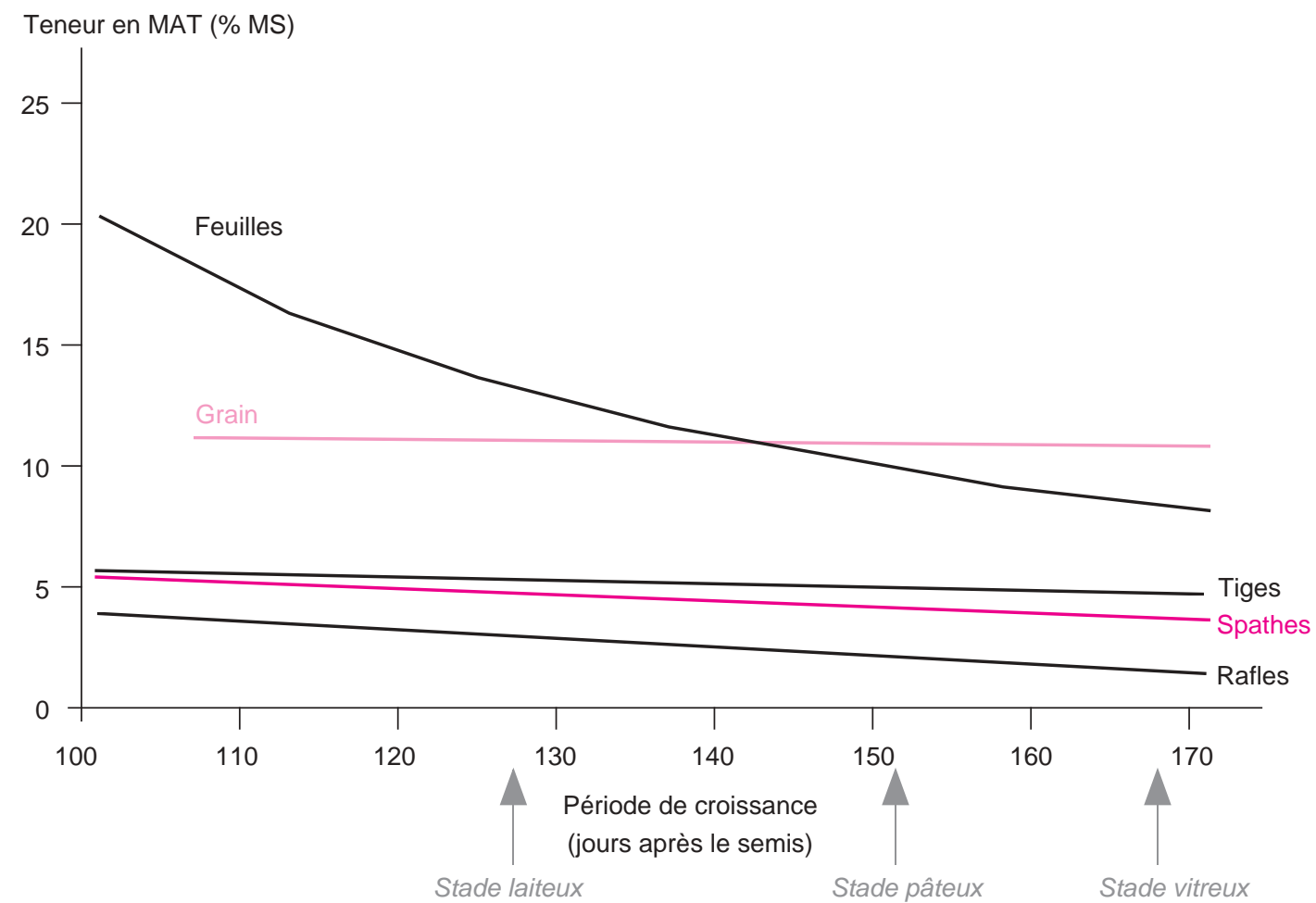


effet de la variété sur la teneur en MAT des maïs pourrait avoir plusieurs origines. Des différences de stade de maturité, liées à des différences de précocité entre variétés, peuvent peut-être expliquer une partie de ces variations comme nous l'avons vu précédemment. L'évolution des bases génétiques pourrait avoir conduit à des teneurs en MAT intrinsèquement plus faibles ou encore l'aug-

Figure 5. Contribution des différents organes $d u$ maïs à la teneur en MAT de la plante entière au stade ensilage (d'après Kuehn et al 1999).

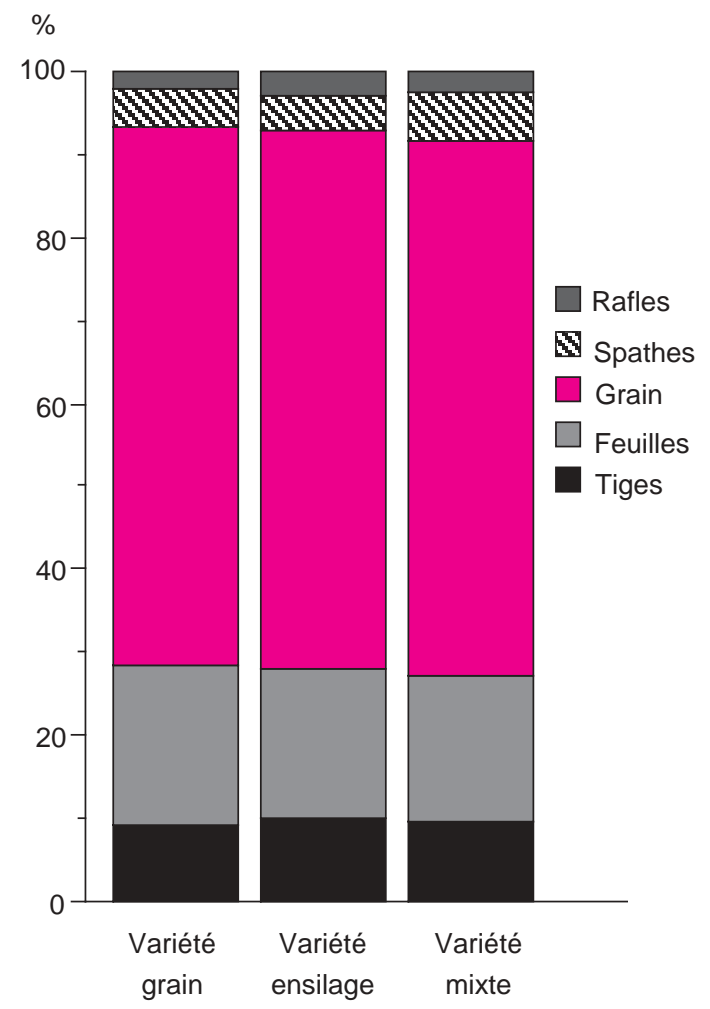

mentation de la productivité des variétés en relation avec les progrès de la sélection a pu se traduire par une dilution de l'azote dans la plante.

Pour tenter d'expliquer ces variations, nous avons calculé, au sein de ces sous-ensembles, les relations entre la teneur en MAT d'une part et les autres critères de la base, à savoir le rendement, mais aussi les teneurs en amidon et en parois qui sont des indicateurs de la composition morphologique de la plante, et la digestibilité-cellulase, critère de prévision de la valeur énergétique des maïs. Une augmentation du rendement tend à diminuer la teneur en MAT de la plante de maïs, mais cette relation est significative seulement dans $25 \%$ des analyses. Quand cette relation est significative, une augmentation entre années du rendement de $0,15 \mathrm{t}$ MS/ha entraîne en moyenne une diminution de la teneur en MAT de 0,03 point. Ces résultats sont en accord avec ceux obtenus récemment par Barrière $e t$ al (2003) à Lusignan, à savoir qu'une variabilité génétique semble bien exister pour la teneur en MAT des maïs, et qu'elle est pour partie indépendante de la productivité. Nous n'avons pas observé de relations significatives entre les teneurs en MAT et les autres constituants chimiques de la plante, amidon et parois, ce qui laisse supposer que les variations de teneur en MAT entre variétés ne sont pas liées à une modification de la composition morphologique de la plante. Au cours de deux années d'expérimentation, Andrieu et al (1993) ont comparé cinq variétés de maïs sur deux localisations différentes, à ClermontFerrand et à Lusignan. A un même stade de maturité et au sein d'un même milieu, les variations de teneur en MAT entre hybrides étaient statistiquement indépendantes de la teneur en grain de la plante (figure 6). Enfin, les corrélations entre la teneur en MAT et la

Figure 6. Effet de la variété sur la teneur en MAT du maïs plante entière au moment de la récolte (d'après Andrieu et al 1993). Au sein d'un même milieu (lieu x année), les points correspondent à des variétés différentes.

Teneur en MAT (\% MS)

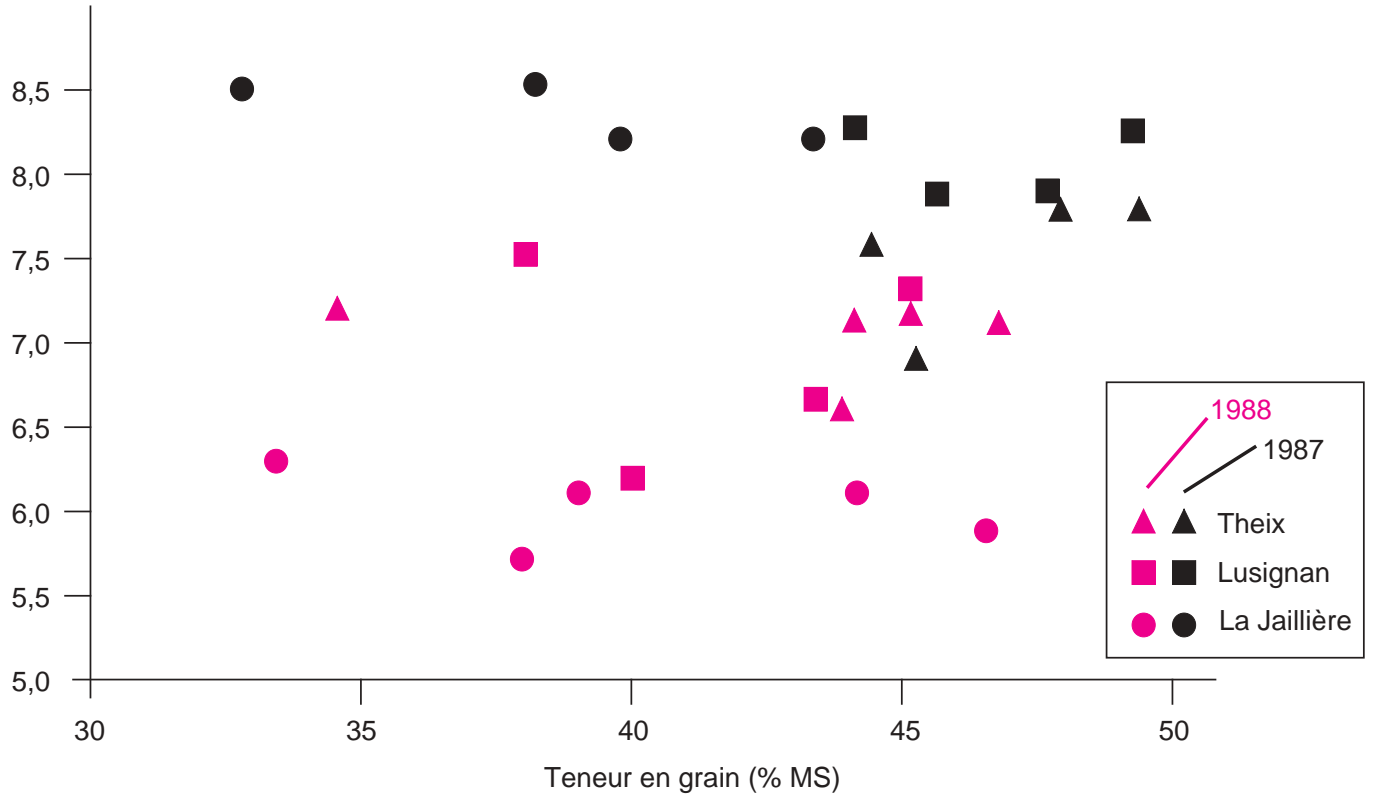


digestibilité de la plante ne sont significatives que pour 22 milieux sur 203, soit dans $11 \%$ des cas, et les coefficients des régressions se distribuent autour de 0. Dans le futur, il semblerait donc qu'il soit possible de progresser en terme de sélection génétique sur les différents paramètres de la valeur nutritive des maïs, à la fois la digestibilité et la teneur en MAT.

Par ailleurs, une des questions auxquelles nous souhaitions répondre à travers cette étude portait sur l'évolution de la teneur en MAT des maiis. En effet des teneurs en MAT faibles ou très faibles sont régulièrement rapportées par les éleveurs. La teneur en MAT des maïs serait passée dans certains cas de valeurs voisines de 8 à $9 \%$ à des valeurs plus proches de $6 \%$ en cinq ans (Barrière et al 2003). Pour appréhender cette diminution et le rôle éventuel de la sélection végétale dans cette évolution, nous avons constitué quatre classes regroupant les variétés en fonction de leur année d'inscription, entre 1980 et 2000, et nous avons sélectionné les sous-ensembles « variété » dans lesquelles ces quatre classes étaient représentées. Les variétés déposées entre 1991 et 1995 ont une teneur en MAT significativement plus faible que celle des variétés déposées les années précédentes, mais cette diminution n'est que de 0,4 point entre les variétés des années 80 et celles des années 90 (figure 7). De plus, ces résultats sont potentiellement biaisés par un certain nombre d'éléments : les effectifs entre classes sont très différents, la classe des variétés déposées entre 1991 et 1995 représentant plus de $90 \%$ des données ; les variétés déposées ne sont pas toujours retenues à l'inscription et ne reflètent donc pas la variabilité génétique réellement utilisée.

Il existe donc une variabilité génétique de la teneur en MAT des maïs plante entière au moment de la récolte. Ces variations dépendent partiellement du rendement, mais ne sont liées ni à la teneur en grain, ni à la diges-

Figure 7. Evolution de la teneur en MAT des maïs plantes entières en fonction de la date de dépôt des variétés pour leur inscription.

Teneur en MAT (\% MS)

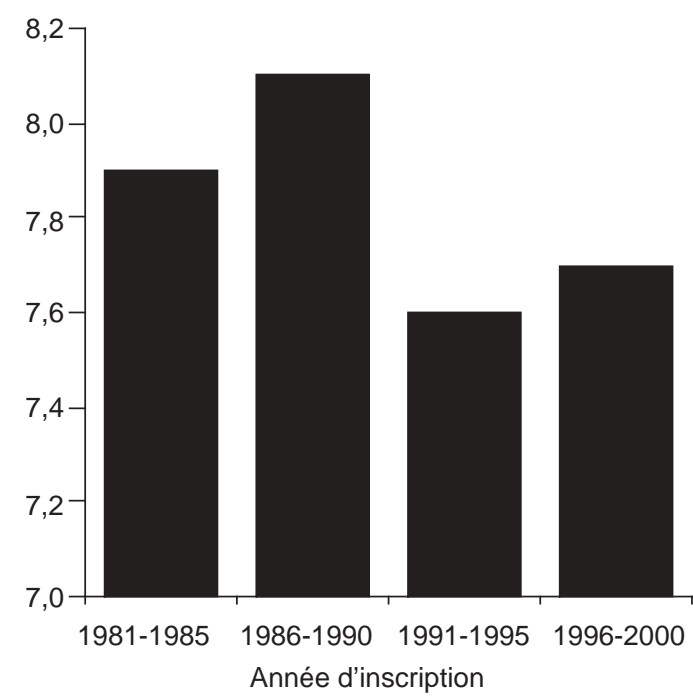

tibilité, ce qui laisse entrevoir des possibilités de sélection spécifique sur les différentes composantes de la valeur nutritive et en particulier la teneur en MAT.

\section{4 / Teneur en MAT et conditions de milieu}

L'expression du milieu peut être considérée comme la combinaison des effets du lieu d'implantation de la culture et de son année d'implantation. Ce facteur, difficile à contrôler, semble pourtant responsable d'une large part des variations de la teneur en MAT de la plante.

Nous avons étudié l'effet du milieu dans 23 sous-ensembles correspondant chacun à une variété cultivée dans plus de 20 milieux différents. Cet effet est significatif dans plus de $80 \%$ des analyses, et explique en moyenne $79 \%$ des variations observées. Pour une même variété, l'étendue de la variation de la teneur en MAT est en moyenne élevée, de l'ordre de 3,4 points, et l'écart type moyen pondéré lié au milieu est en moyenne de 0,65 point, soit une variabilité supérieure à celle observée au niveau de la variété.

Comme précédemment, pour tenter d'expliquer ces variations entre milieux, nous avons calculé au sein de ces sous-ensembles les relations entre la teneur en MAT d'une part et les autres critères de la banque, à savoir le rendement, les autres caractéristiques de la composition chimique, matières minérales, amidon et parois, et la digestibilité. Les variations de productivité entre maïs sous l'effet des caractéristiques du milieu ne sont que peu liées aux variations de teneur en MAT, la relation n'étant significative que dans 8 sousensembles sur 23 , soit $35 \%$ des cas, d'où une certaine indépendance entre ces deux critères. Par ailleurs, les modifications de composition morphologique que traduisent les variations de teneur en amidon et en parois entre différents milieux (et au sein d'une même variété), sont sans effet sur la teneur en MAT des maïs. Ces résultats sont en accord avec les conclusions des travaux déjà anciens de Frölich et al (1980), pour qui les variations de teneur en MAT de la plante de maïs en fonction des conditions de milieu s'expliquent non pas par des modifications de la composition morphologique, mais par les variations de la teneur en MAT de la tige. Une relation positive et significative dans $50 \%$ des cas (12 sous-ensembles sur 23) a été trouvée intra-variété entre les teneurs en MAT et en matières minérales (figure 8). Une modification des conditions de culture par la nature du sol, le niveau de fertilisation, l'irrigation ... induirait donc une réponse comparable en terme de teneur en matières minérales et en azote dans la plante, soit une évolution parallèle des prélèvements de la plante vis-à-vis de ces deux constituants. Enfin, les relations entre la teneur en MAT et la digestibilité de la plante ont été négatives et significatives dans $50 \%$ des cas (12 sous-ensembles sur 23). Dans ces 12 sous-ensembles, une augmentation de 1 point de la digestibilité correspond en
Une grande partie des variations de teneur en MAT s'explique par les conditions de milieu. 
Figure 8. Relations entre les teneurs en matières minérales et en matières azotées de deux variétés cultivées respectivement dans 163 (symboles rouges) et 20 (symboles noirs) milieux différents.

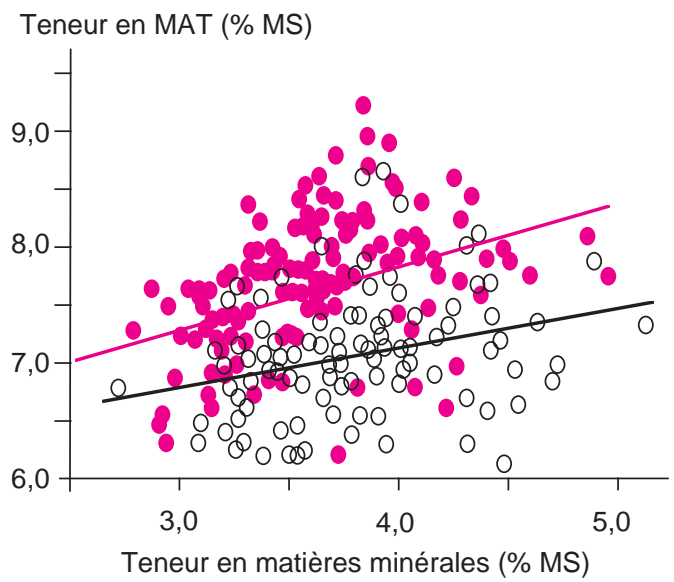

moyenne à une diminution de la teneur en MAT de 0,08 point. Toutefois, pour expliquer cette liaison statistique négative entre digestibilité et teneur en MAT, il faudrait pouvoir analyser séparément l'effet du lieu et de l'année. Les quelques données dont nous disposons ne vont pas dans le sens de cette liaison négative. Ainsi, les mêmes trois hybrides cultivés la même année en deux lieux différents, l'un en conditions normales de végétation et l'autre en conditions séchantes, ont eu à la fois une teneur en MAT et une digestibilité in vivo supérieures dans le lieu correspondant aux conditions normales (voir aliments $n^{\circ} 427$ et 432 de la table INRA, Andrieu et al 1988). Dans le travail mené par le «club digestibilité » (Andrieu et al 1993), les mêmes hybrides $(\mathrm{n}=17)$ ont été étudiés dans les mêmes lieux $(n=4)$ au cours de deux années successives. Les résultats de ces deux années ne font pas apparaître de liaison entre les teneurs en MAT et en matières minérales et font apparaître une relation positive $\left(R^{2}=0,354\right)$ entre la teneur en MAT et la digestibilité in vivo.

\section{5 / Effet de l'ensilage sur la teneur en MAT}

La banque de données dont nous disposions ne permettait pas d'aborder l'effet de l'ensilage sur la teneur en MAT du fourrage, toutes les données étant obtenues au moment de la récolte, nous avons donc étudié les effets de ce facteur à travers les résultats de la littérature.

Alors que la concentration de certains constituants du maiis diminue, notamment la concentration des glucides solubles, la teneur en MAT reste relativement stable (Danley et Vetter 1973, Lessard et al 1978, Phipps et Buchanan-Smith 1981), voire augmente un peu (Andrieu et Demarquily 1974, Wilkinson et Phipps 1979). La teneur en MAT de l'ensilage dépend étroitement de celle de la plante sur pied correspondante (Andrieu et Demarquily 1974, Wilkinson et Phibbs 1979). A partir de 35 maïs récoltés en moyenne à $30 \%$ MS, Andrieu et Demarquilly (1974) ont établi une relation très étroite entre la teneur en MAT de l'ensilage (en \% MS) et celle de la plante sur pied correspondante :

MAT ensilage $=0,746$ MAT plante entière $+2,37$ $(\mathrm{N}=35 ; \mathrm{R}=0,897 ; \mathrm{ETR}=0,7)$

Cela se traduit par une augmentation moyenne de 0,40 point de la teneur en MAT de l'ensilage par rapport à celle de la plante sur pied. Cette augmentation passive traduit essentiellement le fait que les pertes de MAT lors du processus d'ensilage (jus, gaz) ou du séchage à l'étuve (produits volatils) sont plus faibles que celles du reste de la matière sèche. Cette augmentation porte essentiellement sur la teneur en MAT de la fraction canne (Russell 1986), probablement du fait des teneurs en MS différentes des fractions de la plante. Pour une plante entière à $37 \% \mathrm{MS}$, la teneur en MS de l'épi est en moyenne de $51 \%$ et celle de la tige de $24 \%$ (Kuehn et al 1999). La fraction canne subirait donc des pertes en jus plus importantes que la fraction grain.

Sur le plan qualitatif, le processus d'ensilage induit d'importantes modifications de la composition de cette fraction azotée. La teneur en azote soluble, exprimée en proportion de l'azote total, passe en moyenne de $26 \%$ pour le fourrage sur pied à $47 \%$ pour le fourrage ensilé, et cette augmentation est d'autant plus importante que la teneur en MS

Figure 9. Modifications de la composition des matières azotées du maïs au cours de l'ensilage (d'après Andrieu et Demarquilly 1974).

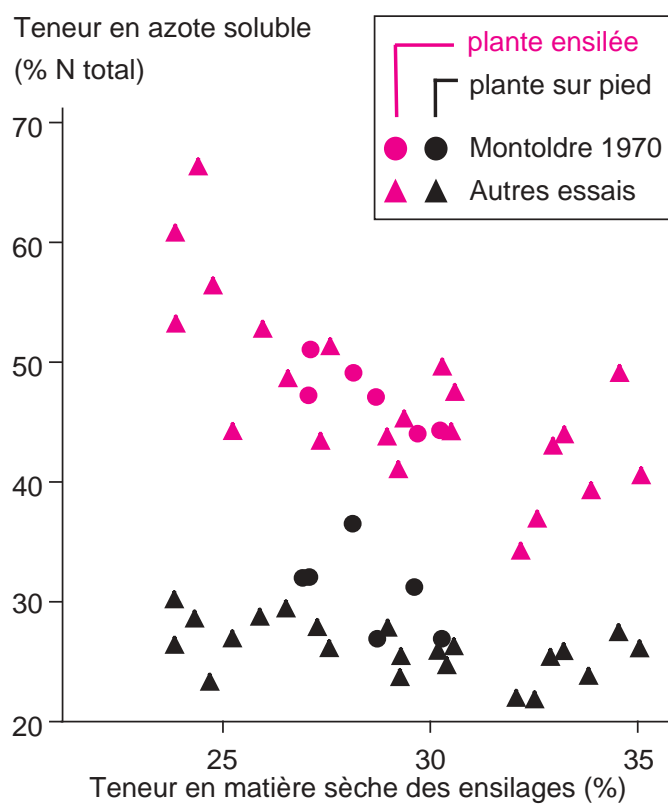

Teneur en azote ammoniacal

des ensilages ( $\% \mathrm{~N}$ total)

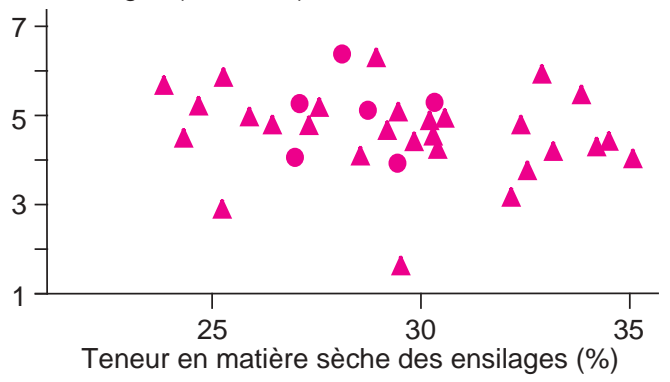


de l'ensilage est faible, et donc que le stade de maturité est précoce (figure 9). La proportion d'azote sous forme ammoniacale reste faible, $5 \%$ en moyenne, et varie dans des proportions relativement étroites, de 2 à $6 \%$ (figure 9). La proportion d'ammoniac est indépendante de la teneur en MS de l'ensilage, mais diminue significativement quand le $\mathrm{pH}$ augmente (Andrieu et Demarquilly 1974) ; la formation d'ammoniac résulterait essentiellement des fermentations donnant naissance aux acides lactique et acétique. Cette modification de la composition biochimique de la fraction azotée du maïs va se traduire sur le plan nutritionnel par une augmentation de la vitesse de dégradation dans le rumen des maïs ensilés par rapport aux maïs au moment de la mise en silo.

\section{Conclusions et perspectives}

Dans la banque de données que nous avons analysée, les génotypes testés correspondent au sous-ensemble de variétés répondant aux critères d'inscription au catalogue français. Ils ne représentent donc pas toute la variabilité génétique pouvant exister au sein de l'espèce maïs. De plus les conditions de milieu étudiées correspondent essentiellement à celles des zones du Nord-Ouest de la France à climat océanique. Il convient donc d'être pru- dent dans la généralisation des corrélations et des liaisons observées dans cette étude. La teneur en MAT des maïs au moment de la récolte varie dans des proportions importantes, du simple au double, et la variabilité liée aux conditions de milieu est supérieure à la variabilité génotypique. La relation négative observée dans un certain nombre d'analyses entre teneur en MAT et rendement traduirait un effet négatif indirect de la sélection pour d'autres caractères (productivité, résistance à la verse ...), mais cet effet n'est pas systématique. L'absence de relation génotypique entre teneur en MAT et digestibilité semble ouvrir des perspectives intéressantes de sélection sur ces deux critères.

Par ailleurs, il est important de noter l'importance des modifications de la composition biochimique des protéines au cours du processus d'ensilage, du fait de leur rôle dans la vitesse de dégradation dans le rumen et donc dans la détermination de la valeur azotée des maiis. Mais contrairement aux autres fourrages, on connaît mal les facteurs de variation de la dégradabilité du maïs, qu'il s'agisse de la plante au moment de la récolte ou ensilée. Or la dégradabilité du maiis dans le rumen étant relativement faible, une variation même faible de la vitesse de dégradation devrait se traduire par une modification non négligeable de sa valeur azotée pour l'animal.

\section{Références}

Aerts J.V., de Brabander D.L., Cottyn B.G., Boucque Ch.V., Buysse F.X., 1976. Evolution de la composition, de la digestibilité et du rendement du maïs en fonction du stade de maturité. Rev. Agric., 2, 379-430.

Andrieu J., Demarquilly C., 1974. Valeur alimentaire du maïs fourrage. III - Influence de la composition et des caractéristiques fermentaires sur la digestibilité et l'ingestibilité des ensilages de maïs. Ann. Zootech., 23, 27-43.

Andrieu J., Demarquilly C., Sauvant D., 1988. Tables de la valeur nutritive des aliments. In : R. Jarrige (ed), Alimentation des Bovins, Ovins, Caprins, 356-461. INRA Editions, Paris.

Andrieu J., Demarquilly C., Dardenne P., Barrière Y., Lila M., Maupetit P., Rivière F., Femenias N., 1993. Composition and nutritive value of whole maize plants fed fresh to sheep. I. Factors of variation. Ann. Zootech., 42, 221-249.

Aufrère J., Michalet-Doreau B., 1988. Comparison of methods for predicting digestibility of feeds. Anim. Feed Sci. Technol., 20, 203-218.

Bal M.A., Coors J.G., Shaver R.D., 1997. Impact of the maturity of corn for use as silage in the diets of dairy cows on intake, digestion, and milk production. J. Dairy Sci., 80, 2497-2503.

Barrière Y., Emile J.C., Surault F., 2003. Variabilité génétique de la teneur en matières azotées du maïs fourrage. Fourrages, 174, 277-284.

Danley M.M., Vetter R.L., 1973. Changes in carbohydrate and nitrogen fraction and digestibility of forages: method of sample processing. J. Anim. Sci., 37, 994-999.

Frölich W.G., Pollmer W.G., Klein D., 1980. Dry matter accumulation in maïze hybrids diverse for protein content under different western european environments. In : Pollmer W.G., Phipps R.H. (eds), Improvement of quality traits of maïze for grains or silage use, 199-200. The Hague, Boston, London.
Huber J.T., Grap G.C., Engel R.W., 1965. Effect of maturity on nutritive value of corn silage for lactating cows. J. Dairy Sci., 48, 1121-1123.

Johnson R.R., McClure K.E., 1968. Corn plant maturity. IV. Effects on digestibility of corn silage in sheep. J. Anim. Sci., 27, 535-540.

Kuehn C.S., Linn J.G., Johnson D.G., Jung H.G., Endres M.I., 1999. Effect of feeding silages from corn hybrids selected for leafiness or grain to lactating dairy cattle. J. Dairy Sci., 82, 2746-2755.

Lessard J.R., Erfle J.D., Sauer F.D., Mahadevan S., 1978 Protein and free amino-acid patterns in maize ensiled with or without urea. J. Sci. Food Agric., 29, 506-512.

Montgomery M.J., Fribourg H.A., Overton J.R., Hoper W.M., 1974. Effect of maturity on silage quality and milk production. J. Dairy Sci., 57, 698-703.

Phipps R.H., Buchanan-Smith J.G., 1981. Effects of ensiling upon free amino-acids and amines in whole plant corn, and on its subsequent nutritive value for lambs. Can. J. Anim. Sci., 62, 259-267.

Phipps R.H., Sutton J.D., Beever D.E., Jones A.K., 2000 The effect of crop maturity on the nurtional value of maize silage for lactating dairy cows. 3. Food intake and milk production. Anim. Sci., 71, 401-409.

Russell J.R., 1986. Influence of harvest date on the nutritive value and ensiling characteristics of maïze. Anim. Feed Sci. Technol., 14, 11-27.

Thomson A.J., Rogers H.H., 1968. Yield and quality components in maize grown for silage. J. Agric. Sci., Camb., 71, 393-403.

Wilkinson J.M., Phipps R.H., 1979. The development of plant components and their effects on the composition of fresh and ensiled forage maïze. J. Agric. Sci., Camb., 92, 483-491. 


\begin{abstract}
Variability and factors of variation of crude protein content in whole maize plant.

We used a database containing 16368 data obtained on whole plant maize, and literature data to evaluate the extent and factors of variation of crude protein (CP) content. CP content of whole plant maize at harvest was $7.7 \pm 0.7 \% \mathrm{DM}$ on average, and varied between 4.7 and $10.5 \%$ DM. CP and ashes contents were significantly correlated, involving a relationship between $\mathbf{N}$ and minerals absorption by plant. These variations in CP content had several causes. It decreased to a low extent with maturity stage, 0.20 percentage unit between dough-flint and flint stages. Variability due to "milieu" (location $\mathrm{x}$ year) was higher than variability
\end{abstract}

Abstract

due to variety (standard deviation of 0.65 and 0.47 percentage unit, respectively). The negative relationship between CP content and yield sometimes observed might express an indirect negative effect of selection on maize CP content, but this relationship was not systematic. Ensiling involved a passive increase in $\mathrm{CP}$ content ( 0.40 percentage unit) with large modifications of biochemical composition of proteins.

MICHALET-DOREAU B., CORNELOUP F., AIZAC B., ANDRIEU J., BAUMONT R., 2004. Variabilité et facteurs de variation de la teneur en matières azotées des maïs récoltés en plantes entières. INRA Prod. Anim., 17, 3-10. 